\title{
Genetic polymorphism of HPA1-17 alloantigen system in the Achang and Jingpo populations population in Yunnan
}

\author{
Weiqun Dong, Dongmei Wang, Yi Li, Sui Wu \\ Department of Blood Transfusion, First Affiliated Hospital of Kunming Medical University, Kunming 650032, China \\ Contributions: (I) Conception and design: W Dong, S Wu; (II) Administrative support: W Dong; (III) Provision of study materials or patients: \\ W Dong, D Wang, Y Li; (IV) Collection and assembly of data: All authors; (V) Data analysis and interpretation: W Dong, D Wan, S Wu; (VI) \\ Manuscript writing: All authors; (VII) Final approval of manuscript: All authors. \\ Correspondence to: Sui Wu. No. 160, Palm Tree Camp, Wuhua District, Kunming 650032, China. Email: yoyo7567@163.com.
}

Background: Human platelet alloantigen (HPA) is part of the platelet membrane structure, named HPA117 system. This study aimed to investigate platelet alloimmunity in the Achang and Jingpo population, ethnic minority specific, to establish a typed platelet donor data bank in Yunnan's ethnic minority areas.

Methods: In this study, samples from 139 unrelated healthy cases from the Achang population, 148 cases of the Jingpo population, and 150 healthy cases from Yunnan's Han population were collected as a control. PCR-sequence specific primers (PCR-SSP) methods were respectively adopted to genotype HPA-1-17. The frequency of genes and genotypes was calculated separately, and the frequency distribution of alleles in the Achang population was compared with that of the Han population.

Results: Monomorphic HPA-7-14 and HPA-16 and 17 were found in the samples from the Han population, while HPA-b was not found in any of these samples. In HPA-1, 2, 4, 5, and 6, aa homozygosity was predominant. Monomorphic HPA-7, HPA-9-14 and HPA-16 were found in the samples from the Achang population, while HPA-b was not found. In HPA-1, 2, 4, 5, 6, 8, and 17, aa homozygosity was predominant. The genotype results of the HPA-4, 7, HPA9-12, HPA14, 16-17 antigen systems in the Jingpo population were all aa, while the HPA-b was not detected. In HPA-1, 2, 5, 6, 8 and 13, aa homozygosity was predominant. HPA-3, 15 showed the greatest heterozygosity among the three populations. The frequency of HPA-1a in the Achang population in Yunnan was significantly different from that in the Han population $(\mathrm{P}<0.05)$. There was no difference in the HPA system of the Jingpo population, but the HPA-2a system was different between the Achang and Jingpo populations $(\mathrm{P}<0.05)$.

Conclusions: In Yunnan, the distribution of the allele polymorphism of HPA-1-17 in the Achang and Jingpo population is similarly distributed to that in the Han population and exhibits their own characteristic. Therefore, a database of platelet donor typing of the ethnic group should be established.

Keywords: Human platelet antigens (HPA); ethnic minorities; genotype

Submitted Mar 31, 2020. Accepted for publication Jul 04, 2020.

doi: $10.21037 /$ apm-20-1075

View this article at: http://dx.doi.org/10.21037/apm-20-1075

\section{Introduction}

Many modern clinical studies have noted the relationship between platelet alloimmunity caused by human platelet antigen (HPA) and some diseases, including neonatal allogeneic thrombocytopenia (NATP), ineffective platelet transfusion (PRT), post-transfusion purpura
(PTP), transplant-related thrombocytopenia, and arterial thrombotic diseases $(1,2)$. The distribution frequency of HPA antigens among different populations bears a positive relationship with the likelihood of developing HPA immunity. In this study, the Achang and Jingpo population, ethnic groups unique to Yunnan province, were selected as the research object, and the Han ethnic group in 
Yunnan was used as the control. The most commonly used HPA genotyping technique of PCR-SSP was employed, and a comparative analysis of the HPA gene distribution and genotype frequencies in the three populations was made, to explore the relationship between platelet antigen polymorphisms and regions, as well as ethnicities. A platelet gene database for blood resources in Yunnan's minority regions, which helps to explore the origin, inheritance, migration of ethnic minorities and provide data for forensic individual identification, should be established.

\section{Methods}

\section{Research subjects}

Samples from 139 healthy cases were collected from the Achang population, 148 healthy cases from the Jingpo population in the south of Yunnan province, China, and samples from 150 healthy cases were collected from the Han population in Kunming, Yunnan as a control. Each sample was traced back for three generations to ensure there was no history of intermarriage with other ethnic groups. All of the selected subjects were ethically informed and participated on a voluntary basis. The study was approved by First Affiliated Hospital of Kunming Medical University (No. KMML12011122) and written informed consent was obtained from all patients. The study was conducted in accordance with the Declaration of Helsinki (as revised in 2013).

\section{Specimen collection}

A total volume of 2 to $3 \mathrm{~mL}$ venous blood of anticoagulant EDTA-K2 was collected from each participant, and frozen at $-80^{\circ} \mathrm{C}$ for later examination.

\section{Instruments and reagents}

The PCR instrument and horizontal electrophoresis system were purchased from Bio-Rad Corporation (California, USA). The Gel Doc EQ Imaging System was obtained, also from Bio-Rad Corporation (USA), for gel imaging analysis. A platelet antigen genotyping kit was obtained from Changchun Bode Biotechnology Co., Ltd (China).

\section{DNA extraction and measurement}

The potassium iodide method was applied to measure the ultraviolet absorption peaks of the extracted genomic DNA using a UV spectrophotometer. The absorbance was measured at a wavelength of 260 and $280 \mathrm{~nm}$. The DNA concentration and purity were calculated, and each sample was diluted to a concentration of $1 \mu \mathrm{g} / \mu \mathrm{L}$ and stored at $-20^{\circ} \mathrm{C}$.

\section{HPA genotyping test}

HPA genotyping was conducted using PCR technology and the reaction system included PCR primer mix (specificsequence primer: $0.4 \mu \mathrm{mol} / \mathrm{L}$, internal control primer $0.4 \mu \mathrm{mol} / \mathrm{L}), 10 \times$ PCR Buffer $(5 \mu \mathrm{L})$, dNTP $(0.2 \mu \mathrm{mol} / \mathrm{L})$, Taq enzymes $(0.75 \mathrm{U})$, and genomic DNA $(1 \mu \mathrm{g})$ of HPA1-17, separately. All PCR amplification was performed simultaneously on HPA1 to 17 systems under the same conditions. The PCR reaction conditions were as follows: $94{ }^{\circ} \mathrm{C}$ for $9 \mathrm{~min}$; $\left(94^{\circ} \mathrm{C}, 1 \mathrm{~min} ; 61^{\circ} \mathrm{C}, 1 \mathrm{~min} ; 72{ }^{\circ} \mathrm{C}, 1 \mathrm{~min}\right.$ ) for a total of 35 cycles, and finally prolonged at $72{ }^{\circ} \mathrm{C}$ for $10 \mathrm{~min}$.

\section{Detection of the amplified product}

The PCR product $(10 \mu \mathrm{L})$ was separated in a $2 \%$ agarose gel containing $0.5 \mathrm{mg} / \mathrm{L}$ ethidium bromide, at a voltage of $80 \mathrm{mV}$. After electrophoresis for 30 minutes, the results were recorded. Primer design: the primers of WHO officially named HPA1-17 were provided in the kit. The relative 34 pairs of primers for these genes were designed according to the specific base replacement of HPA-a and HPA-b of the HPA exon specific base, and the specific base was used as the 3 'end of the forward primers for amplification.

\section{Statistical analysis}

Data were analyzed using SAS 9.3 statistical software (North Carolina, USA). Gene frequencies were compared between the different populations using the $\chi^{2}$ test (precise probability method), and the comparison of genotypes was performed by comparing multiple constituent ratios of the $\chi^{2}$ test. $\mathrm{P}<0.05$ was considered to show difference, and $\mathrm{P}<0.01$ was considered to show a significant difference.

\section{Results}

\section{Genetic testing of HPA-1-17 systems and statistical results of the Achang population}

The genetic test results of the HPA-1-17 systems of the 139 cases from the Achang population are shown in Table 1. The PCR results of the HPA-1-17 system genotyping in the Achang population are shown by gel imaging (Figure 1). 
Table 1 Gene and genotype frequencies of HPA1-17 in the Achang population ( $\mathrm{n}=139)$

\begin{tabular}{|c|c|c|c|c|c|c|c|c|c|c|c|c|c|c|c|c|c|}
\hline HPA & \multicolumn{6}{|c|}{ Genotype frequency } & \multicolumn{6}{|c|}{ Hope value } & \multicolumn{2}{|c|}{ Gene frequency } & MP & \multicolumn{2}{|c|}{$\mathrm{H}-\mathrm{W}$ result } \\
\hline HPA-1 & 129 & 92.81 & 10 & 7.19 & 0 & 0.00 & 129.17 & 92.93 & 9.65 & 6.94 & 0.18 & 0.13 & 0.9640 & 0.0360 & 0.0670 & 0.1932 & $>0.05$ \\
\hline HPA-2 & 134 & 96.40 & 5 & 3.60 & 0 & 0.00 & 134.04 & 96.43 & 4.91 & 3.54 & 0.05 & 0.03 & 0.9820 & 0.0180 & 0.0347 & 0.0466 & $>0.05$ \\
\hline HPA-3 & 44 & 31.65 & 67 & 48.20 & 28 & 20.15 & 43.22 & 31.09 & 68.58 & 49.34 & 27.20 & 19.57 & 0.5576 & 0.4424 & 0.3717 & 0.0737 & $>0.05$ \\
\hline HPA-5 & 132 & 94.96 & 7 & 5.04 & 0 & 0.00 & 132.08 & 95.02 & 6.83 & 4.91 & 0.09 & 0.06 & 0.9748 & 0.0252 & 0.0479 & 0.0926 & $>0.05$ \\
\hline HPA-6 & 132 & 94.96 & 7 & 5.04 & 0 & 0.00 & 132.08 & 95.02 & 6.83 & 4.91 & 0.09 & 0.06 & 0.9748 & 0.0252 & 0.0479 & 0.0926 & $>0.05$ \\
\hline HPA-7 & 139 & 100.00 & 0 & 0.00 & 0 & 0.00 & 139.00 & 100.00 & 0.00 & 0.00 & 0.00 & 0.00 & 1.0000 & 0.0000 & 0.0000 & - & - \\
\hline HPA-8 & 138 & 99.28 & 1 & 0.72 & 0 & 0.00 & 138.00 & 99.28 & 1.00 & 0.72 & 0.00 & 0.00 & 0.9964 & 0.0036 & 0.0071 & 0.0018 & $>0.05$ \\
\hline HPA-11 & 139 & 100.00 & 0 & 0.00 & 0 & 0.00 & 139.00 & 100.00 & 0.00 & 0.00 & 0.00 & 0.00 & 1.0000 & 0.0000 & 0.0000 & - & - \\
\hline HPA-12 & 139 & 100.00 & 0 & 0.00 & 0 & 0.00 & 139.00 & 100.00 & 0.00 & 0.00 & 0.00 & 0.00 & 1.0000 & 0.0000 & 0.0000 & - & - \\
\hline HPA-13 & 139 & 100.00 & 0 & 0.00 & 0 & 0.00 & 139.00 & 100.00 & 0.00 & 0.00 & 0.00 & 0.00 & 1.0000 & 0.0000 & 0.0000 & - & - \\
\hline HPA-14 & 139 & 100.00 & 0 & 0.00 & 0 & 0.00 & 139.00 & 100.00 & 0.00 & 0.00 & 0.00 & 0.00 & 1.0000 & 0.0000 & 0.0000 & - & - \\
\hline HPA-15 & 49 & 35.25 & 51 & 36.69 & 39 & 28.06 & 39.93 & 28.73 & 69.14 & 49.74 & 21.53 & 29.93 & 0.5360 & 0.4640 & 0.3737 & 9.5686 & $>0.05$ \\
\hline HPA-16 & 139 & 100.00 & 0 & 0.00 & 0 & 0.00 & 139.00 & 100.00 & 0.00 & 0.00 & 0.00 & 0.00 & 1.0000 & 0.0000 & 0.0000 & - & - \\
\hline HPA-17 & 136 & 97.84 & 3 & 2.16 & 0 & 0.00 & 136.01 & 97.85 & 2.97 & 2.14 & 0.02 & 0.01 & 0.9892 & 0.0108 & 0.0211 & 0.0165 & $>0.05$ \\
\hline
\end{tabular}

HPA, human platelet antigen.

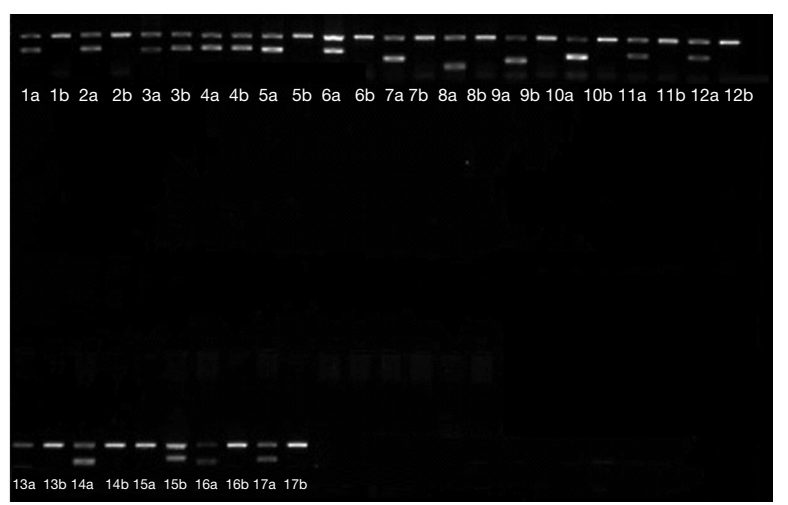

Figure $1 \mathrm{HPA}$ gel image of sample No. 122 from the Achang group. Note: HPA 1aa, 2aa, 3ab, 4ab, 5aa, 6aa, 7aa, 8aa, 9aa, 10aa, $11 \mathrm{aa}, 12 \mathrm{aa}, 13 \mathrm{aa}, 14 \mathrm{aa}, 15 \mathrm{bb}, 16 \mathrm{aa}, 17 \mathrm{aa}$. HPA, human platelet antigen.

The results showed that aa homozygosity was the only genotype of the HPA-7, HPA-9-14, and HPA-16 antigen systems, while no corresponding alleles of HPA-b were found. The predominant genotype of the antigen systems in HPA-1, 2, 4, 5, 6, 8, 17 was aa, although HPA-3, 15 showed a greater degree of heterozygosity. For HPA-3, the genotype frequencies of aa, $3 \mathrm{ab}$, and $3 \mathrm{bb}$ were $31.65 \%$, $48.2 \%$, and $20.15 \%$, respectively. Meanwhile, for HPA15 , the genotype frequencies of aa, $15 \mathrm{ab}$, and $15 \mathrm{bb}$ were $35.25 \%, 36.69 \%$, and $28.06 \%$, respectively. After analysis by $\chi^{2}$ test, the results of this genetic test in the Achang population conformed to the $\mathrm{H}-\mathrm{W}$ genetic balance law.

\section{Genetic testing of HPA-1-17 systems and statistical results of the Fingpo population}

Results of HPA1-17 system genetic testing of 148 cases from the Jingpo population are shown in Table 2. And the gel images of HPA1-17 system genotyping in the Jingpo population are shown in Figure 2.

The genotypes of the HPA-4, 7, HPA9-12, HPA14, 16-17 antigen systems in the Jingpo population were all $\mathrm{aa}$, and the corresponding allele HPA-b was not detected; 
Table 2 Gene and genotype frequencies of HPA1-17 in the Jingpo population ( $\mathrm{n}=148)$

\begin{tabular}{|c|c|c|c|c|c|c|c|c|c|c|c|c|c|c|c|c|c|}
\hline HAP & \multicolumn{6}{|c|}{ Genotype frequency } & \multicolumn{6}{|c|}{ Hope value } & \multicolumn{2}{|c|}{ Gene frequency } & MP & \multicolumn{2}{|c|}{$\mathrm{H}-\mathrm{W}$ result } \\
\hline HPA-1 & 143 & 96.62 & 5 & 3.38 & 0 & 0.00 & 143.04 & 96.65 & 4.92 & 3.32 & 0.04 & 0.03 & 0.9831 & 0.0169 & 0.0327 & 0.0437 & $>0.05$ \\
\hline HPA-2 & 127 & 85.81 & 21 & 14.19 & 0 & 0.00 & 127.76 & 86.32 & 19.50 & 13.17 & 0.74 & 0.50 & 0.9291 & 0.0709 & 0.1230 & 0.8641 & $>0.05$ \\
\hline HPA-3 & 55 & 37.16 & 73 & 49.32 & 20 & 13.52 & 56.56 & 38.22 & 69.86 & 47.21 & 21.57 & 14.58 & 0.6182 & 0.3818 & 0.3606 & 0.2987 & $>0.05$ \\
\hline HPA-5 & 140 & 94.59 & 8 & 5.41 & 0 & 0.00 & 140.12 & 94.67 & 7.78 & 5.25 & 0.11 & 0.07 & 0.9730 & 0.0270 & 0.0512 & 0.1144 & $>0.05$ \\
\hline HPA-6 & 146 & 98.65 & 2 & 1.35 & 0 & 0.00 & 145.99 & 98.64 & 2.00 & 1.35 & 0.01 & 0.00 & 0.9932 & 0.0068 & 0.0134 & 0.0068 & $>0.05$ \\
\hline HPA-7 & 148 & 100.00 & 0 & 0.00 & 0 & 0.00 & 148.00 & 100.00 & 0.00 & 0.00 & 0.00 & 0.00 & 1.0000 & 0.0000 & 0.0000 & - & - \\
\hline HPA-8 & 147 & 99.32 & 1 & 0.68 & 0 & 0.00 & 147.00 & 99.32 & 1.00 & 0.68 & 0.00 & 0.00 & 0.9966 & 0.0034 & 0.0068 & 0.0017 & $>0.05$ \\
\hline HPA-11 & 148 & 100.00 & 0 & 0.00 & 0 & 0.00 & 148.00 & 100.00 & 0.00 & 0.00 & 0.00 & 0.00 & 1.0000 & 0.0000 & 0.0000 & - & - \\
\hline HPA-12 & 148 & 100.00 & 0 & 0.00 & 0 & 0.00 & 148.00 & 100.00 & 0.00 & 0.00 & 0.00 & 0.00 & 1.0000 & 0.0000 & 0.0000 & - & - \\
\hline HPA-13 & 147 & 99.32 & 1 & 0.68 & 0 & 0.00 & 147.00 & 99.32 & 1.00 & 0.68 & 0.00 & 0.00 & 0.9966 & 0.0034 & 0.0068 & 0.0017 & $>0.05$ \\
\hline HPA-14 & 148 & 100.00 & 0 & 0.00 & 0 & 0.00 & 148.00 & 100.00 & 0.00 & 0.00 & 0.00 & 0.00 & 1.0000 & 0.0000 & 0.0000 & - & - \\
\hline HPA-15 & 42 & 28.38 & 82 & 55.41 & 24 & 16.21 & 46.55 & 31.45 & 72.91 & 49.26 & 28.55 & 19.29 & 0.5608 & 0.4392 & 0.3713 & 2.3031 & $>0.05$ \\
\hline HPA-16 & 148 & 100.00 & 0 & 0.00 & 0 & 0.00 & 148.00 & 100.00 & 0.00 & 0.00 & 0.00 & 0.00 & 1.0000 & 0.0000 & 0.0000 & - & - \\
\hline HPA-17 & 148 & 100.00 & 0 & 0.00 & 0 & 0.00 & 148.00 & 100.00 & 0.00 & 0.00 & 0.00 & 0.00 & 1.0000 & 0.0000 & 0.0000 & - & - \\
\hline
\end{tabular}

HPA, human platelet antigen.

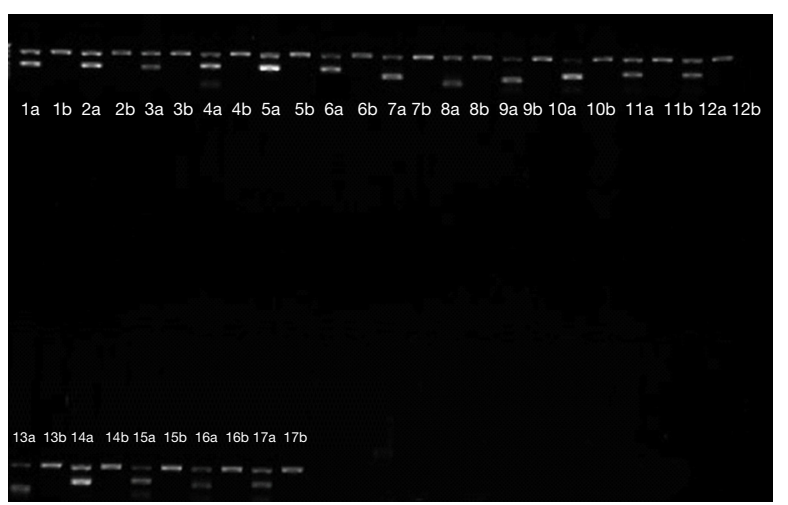

Figure 2 HPA gel image of sample No. 41 from the Jingpo group. Note: HPA 1aa, 2aa, 3aa, 4aa, 5aa, 6aa, 7aa, 8aa, 9aa, 10aa, 11aa, 12aa, 13aa, 14aa, 15aa, 16aa, 17 aa.

The genotype of HPA-1, 2, 5, 6, 8 and 13 antigen systems were mostly aa, and HPA- 3 and 15 showed a high degree of heterozygosity. The genotype frequencies of HPA-3aa, $3 \mathrm{ab}$ and $3 \mathrm{bb}$ were $37.16 \%, 49.32 \%, 13.52 \%$, and the genotype frequencies of HPA-15aa, $15 \mathrm{ab}$ and $15 \mathrm{bb}$ were $28.38 \%$, $55.41 \%$ and $16.21 \%$, respectively. According to $\chi^{2}$ test, the genetic test results of the Jingpo population samples conformed to the $\mathrm{H}-\mathrm{W}$ genetic balance law.

\section{Distribution of HPA-1-17 system genes and genotype frequencies in the Han population}

In the HPA-7-14, HPA-16, and HPA-17 antigen systems, aa was the only genotype in the 150 Han population, and the corresponding allele HPA-b was not detected (Figure 3). In HPA-1, 2, 4, 5, and 6 antigen systems, aa was also the predominant genotype. The genotypes of HPA-3 and 15 had the same high degree of heterozygosity, and the genotype frequencies of aa, ab, and bb were 34\%, 46.67\% and $1933 \%$, respectively (Table 3). 


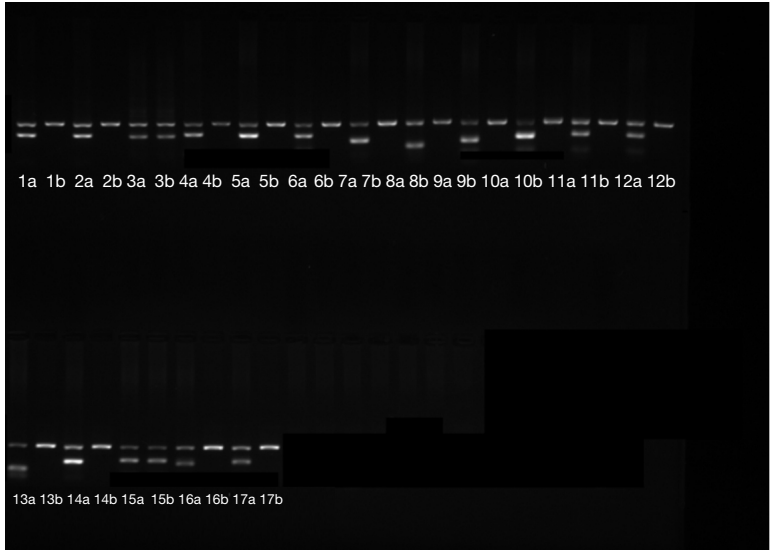

Figure 3 HPA gel image of sample No. 8 from the Han group. Note: HPA 1aa, 2aa, 3ab, 4aa, 5aa, 6aa, 7aa, 8aa, 9aa, 10aa, 11aa, 12aa, 13aa, 14aa, 15ab, 16aa, $17 \mathrm{aa}$.

\section{Comparison of gene frequency between the Achang, Fingpo and Han populations}

The genotype was aa in the HPA-7, HPA9-12, HPA-14, 16 systems of the three populations, and the corresponding allele HPA-b was not detected. High polymorphism was found in HPA-3 and HPA-15 of these three ethnic groups. The HPA-1a system in the Achang group was significantly different from that of the Han population, and the HPA2a system is significantly different between the Achang and Jingpo populations $(\mathrm{P}<0.05$, Table 4).

\section{Discussion}

Platelet alloimmunity caused by HPA has been shown

Table 3 Gene and genotype frequencies of HPA1-17 in the Han population $(n=150)$

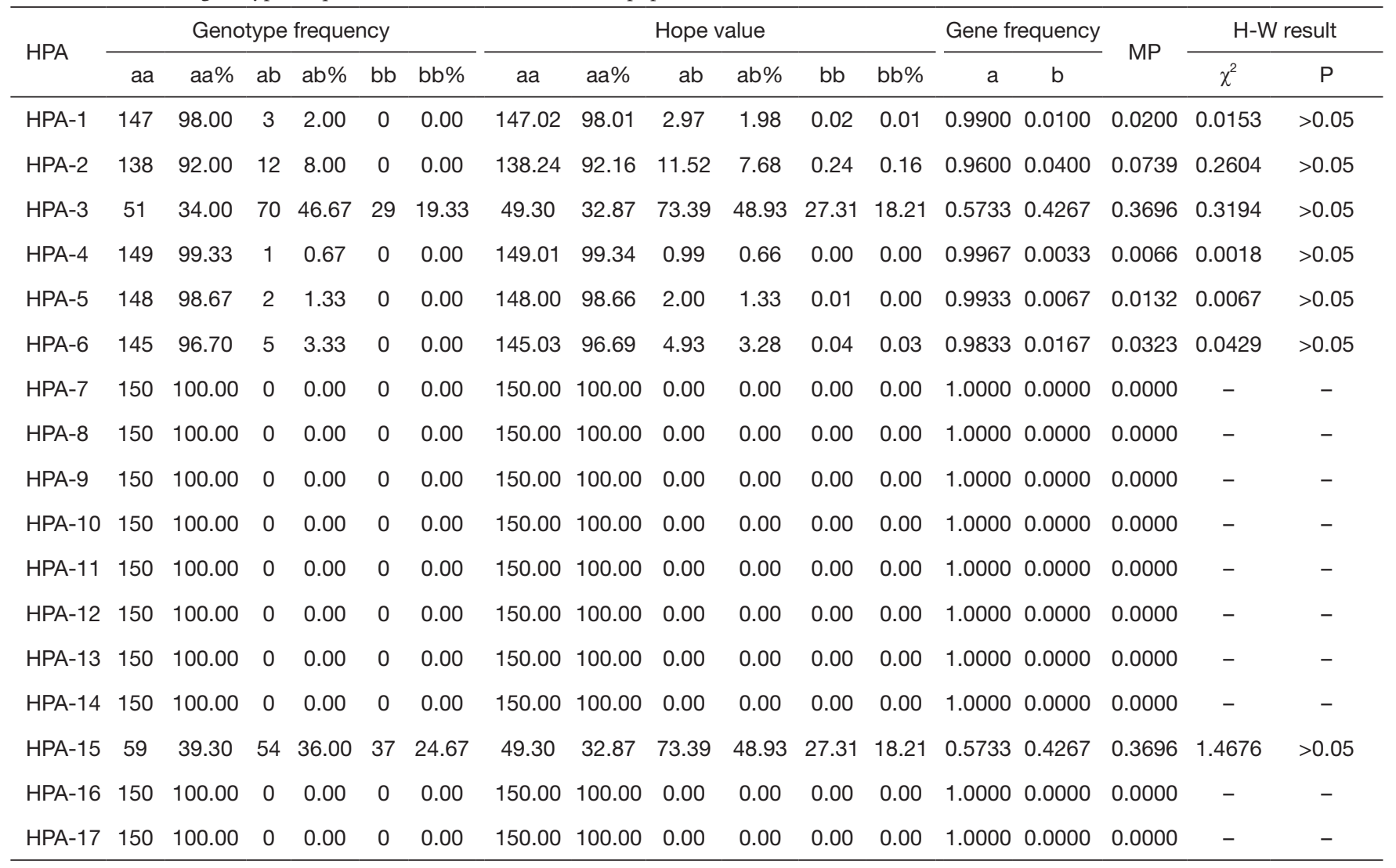

HPA, human platelet antigen. 
Table 4 Comparison of gene frequencies between the Achang, Jingpo and Han population

\begin{tabular}{|c|c|c|c|}
\hline HPA & Control (Han), $n=150$ & Achang, $n=139$ & Jingpo, $n=148$ \\
\hline HPA-1b & 0.0100 & 0.0360 & 0.0169 \\
\hline HPA-2a & 0.9600 & 0.9820 & $0.9291^{\mathrm{b}}$ \\
\hline HPA-2b & 0.0400 & 0.0180 & 0.0709 \\
\hline HPA-3b & 0.4267 & 0.4424 & 0.3818 \\
\hline HPA-4a & 0.9967 & 0.9964 & 1.0000 \\
\hline HPA-4b & 0.0033 & 0.0036 & 0.0000 \\
\hline HPA-5a & 0.9933 & 0.9748 & 0.9730 \\
\hline HPA-6b & 0.0167 & 0.0252 & 0.0068 \\
\hline HPA-8a & 1.0000 & 0.9964 & 0.9966 \\
\hline HPA-8b & 0.0000 & 0.0036 & 0.0034 \\
\hline HPA-13a & 1.0000 & 1.0000 & 0.9966 \\
\hline HPA-13b & 0.0000 & 0.0000 & 0.0034 \\
\hline HPA-15a & 0.5733 & 0.5360 & 0.5608 \\
\hline HPA-15b & 0.4267 & 0.4640 & 0.4392 \\
\hline HPA-17a & 1.0000 & 0.9892 & 1.0000 \\
\hline
\end{tabular}

Compared with the Han population, ${ }^{a} \mathrm{P}<0.05$, compared with the Achang population, ${ }^{\mathrm{b}} \mathrm{P}<0.05$. HPA, human platelet antigen.

by many recent clinical studies to be related to NATP, ineffective PRT, PTP, transplant-related thrombocytopenia, arterial thrombotic diseases, and other diseases. Therefore, the diagnosis, prevention, and treatment of these diseases need to be based on the genotyping of platelet alloantigens.

Yunnan has the largest ethic minority population of any province, which makes the possibility of blood transfusion between different ethnic minorities exceedingly high. It is therefore necessary to conduct research on HPA gene polymorphisms in ethnic minorities. With the decrease in the ethnic minority population, preserving the ethnogenetic and anthropological data of different ethnic minorities also bears great significance. At present, HPA studies have been conducted at home and abroad, many of which have focused on the Chinese Han population $(3,4)$, while only few investigations into HPA have been performed for ethnic minority groups $(5,6)$, and no reports exist on platelet alloantigens among ethnic minorities in Yunnan province (7).

In this paper, the Achang and Jingpo population, ethnic minority unique to Yunnan, were used as the research object. The HPA gene distribution and genotype frequencies in different populations were analyzed to study the relationship between platelet antigen polymorphisms and area, as well as ethnicity. Genotyping of HPA1-17 indicated that the gene frequencies in the healthy population of Achang and Jingpo people in Yunnan were close to those of Han people in the same area, and they also displayed their own characteristics (Tables 1-4). The HPA-3 and HPA-15 systems of the three populations show high polymorphism, which is obviously higher than other HPA systems. Based on the results of MP value, the HPA-3 and HPA-15 systems were susceptible to the same immune response due to mismatched infusion. The genotypes of the HPA-7, HPA-9-12, HPA-14, 16 systems of the three 
populations were all homozygous aa, and the corresponding allele HPA-b was not detected. The gene frequency of ' $\mathrm{a}$ ' in other HPA systems all exceeded $50 \%$. The HPA-1a gene frequency of the Achang population is significantly different from the Han population, and the HPA-2a system is significantly different between the Achang and Jingpo populations $(\mathrm{P}<0.05)$.

To improve the safety and effectiveness of PRT, attention should therefore be paid to HPA alloimmunization and whether the differences of platelet antigen genes between different nationalities cause different immune effects. According to the frequency of HPA gene polymorphisms in this survey, the risk of platelet alloimmunization in the Achang and Jingpo population can be predicted. In a clinical setting, HPA-1a, HPA-2a, HPA-3, and HPA15 are indicated to be important platelet antigen systems. HPA genotyping of ethnic minorities can provide a basis for the prevention and diagnosis of diseases caused by HPA alloimmunity and the establishment of a platelet donor data bank of ethnic minority area. As a marker of human genetic polymorphism, HPA can also be used in the research of forensic individual identification, human evolution and migration, as well as the correlation analysis of some diseases.

\section{Acknowledgments}

Funding: National Natural Science Foundation of China (81060142) and Applied Basic Research Project of Yunnan Province (2009ZC104M).

\section{Footnote}

Data Sharing Statement: Available at http://dx.doi. org/10.21037/apm-20-1075

Conflicts of Interest: All authors have completed the ICMJE uniform disclosure form (available at http://dx.doi. org/10.21037/apm-20-1075). The authors have no conflicts of interest to declare.

Ethical Statement: The authors are accountable for all aspects of the work in ensuring that questions related to the accuracy or integrity of any part of the work are appropriately investigated and resolved. The study was approved by First Affiliated Hospital of Kunming Medical
University (No. KMML12011122) and written informed consent was obtained from all patients. The study was conducted in accordance with the Declaration of Helsinki (as revised in 2013).

Open Access Statement: This is an Open Access article distributed in accordance with the Creative Commons Attribution-NonCommercial-NoDerivs 4.0 International License (CC BY-NC-ND 4.0), which permits the noncommercial replication and distribution of the article with the strict proviso that no changes or edits are made and the original work is properly cited (including links to both the formal publication through the relevant DOI and the license). See: https://creativecommons.org/licenses/by-nc-nd/4.0/.

\section{References}

1. Santoso S, Kiefel V. Human platelet alloantigens. Wien Klin Wochenschr 2001;113:806-13.

2. Zhao T. Research of human platelet antigen (HPA). Chinese Journal of Blood Transfusion 2004;17:129-32.

3. Chen Y, Ye X, Xia W, et al. Genetic polymorphism of HPA-1 to-17 alloantigen system in Guangzhou population. Chinese Journal of Immunology 2010;26:699-702.

4. Hurd CM, Cavanagh G, Schuh A, et al. Genotyping for platelet-specific antigens. techniques for the detection of single nucleotide polymorphisms. Vox Sang 2002;83:1-12.

5. Xia L, Cai X, Chen X, et al. Study on Gene Polymorphisms of Platelet 1-17 Antigen System in Li People of Hainan Island. Chinese Journal of Medical Genetics 2010;27:229-30.

6. Deng Y, Tan X, Huang J. Differential expression of human platelet antigen gene polymorphisms in Guangxi Zhuang, Yao, Miao, Dai and Dai nationalities. Chinese Journal of Gerontology 2012;32:2499-501.

7. Zhu X, Yang T, Yao F, et al. Gene frequencies of the HLA-A, B and HPA in typed platelet donnors of Yunnan Han Nationality. Journal of Clinical Transfusion and Laboratory Medicine 2012;14:193-200.

Cite this article as: Dong W, Wang D, Li Y, Wu S. Genetic polymorphism of HPA1-17 alloantigen system in the Achang and Jingpo populations population in Yunnan. Ann Palliat Med 2020;9(4):1990-1996. doi: 10.21037/apm-20-1075 